\title{
Commentary
}

\author{
J Moxham
}

The case report by Brander and colleagues of severe diaphragm weakness due to phrenic nerve damage from radiotherapy given many years earlier is of considerable interest. ${ }^{1}$ It provides yet another cause of diaphragm dysfunction to add to an already long list. The authors comment that the potential causes of diaphragm weakness include motor neurone disease, poliomyelitis, myasthenia, muscular dystrophies, polyneuropathy, neuralgic amyotrophy, malignant invasion of the phrenic nerves, chest wall trauma, cardiothoracic surgery, connective tissue diseases, amyloid, and thyroid disease. There are other causes that could be added to this list and it is therefore an important clinical point that diaphragm weakness is unusual, but it is not rare. Any respiratory physician with a busy clinical practice can expect to see patients with diaphragm weakness, of varying severity, on an infrequent but regular basis. An important issue therefore is how diaphragm weakness is diagnosed and how its severity is accurately assessed.

When diaphragm weakness is severe the diagnosis - in a qualitative sense - is not a problem, and the key features, as in this case presentation, are orthopnoea, paradoxical abdominal movement, and a fall in vital capacity when supine. However, these are late symptoms and signs that only occur when diaphragm strength is reduced to approximately $25 \%$ of normal. ${ }^{2}$ From the history of the patient described in this case report it is clear that diaphragm weakness was slowly progressing over several years. Eventually the diagnosis became obvious, but presumably for a prolonged period of time the cause of increasing breathlessness was not appreciated. Until a secure diagnosis of diaphragm weakness is made, breathlessness is likely to be attributed to other factors such as cardiac disease or pulmonary fibrosis, both easily possible in patients treated for malignant disease with radiotherapy and chemotherapy. As an everyday part of clinical practice, respiratory physicians assess breathless patients and conclude that the symptom is due to airways obstruction, obesity, pulmonary oedema, etc, often in combination because we carefully set about the task of assessing the contribution of each of the components. Similarly, we can accurately assess diaphragm strength ${ }^{3}$ and therefore the contribution of diaphragm weakness to breathlessness, but in practice we seldom do so. Most patients with mild through to moderately severe weakness are undiagnosed. How many patients with mild to moderate diaphragm weakness due to one of the many causes listed by Brander and colleagues remain undiagnosed despite diaphragm dysfunction contributing to symptoms of breathlessness and disability? This question can only be answered if we are prepared, when clinically appropriate, to make specific measurements of respiratory muscle strength.

Brander and co-workers treated their patient with nocturnal non-invasive ventilation with good control of symptoms; for patients with severe orthopnoea the achievement of comfortable sleep is most welcome. Such symptomatic control is a good indication for noninvasive ventilation. A second indication would be established chronic ventilatory failure. This did not occur in the case presented because the respiratory muscle weakness was largely confined to the diaphragm. ${ }^{4}$ In patients with more widespread and severe respiratory muscle weakness, ventilatory failure first develops at night (hence the importance of sleep studies) and often occurs rather suddenly when the degree of weakness becomes sufficiently profound. Thus, patients may have a reduction of $30 \%, 40 \%, 50 \%, 60 \%$, or even $70 \%$ in respiratory muscle strength without developing ventilatory failure unless, in addition, there is increased ventilatory load. At a relatively sharp cut off point, when inspiratory muscle strength is approximately $25-30 \%$ of normal, ventilatory failure then becomes quite common. Clearly, if an accurate diagnosis of respiratory muscle weakness at an earlier stage has not been made, the clinician will often be caught unawares by a patient who presents with established ventilatory failure. Not only are such patients at considerable risk of death, but the difficult question of whether to institute ventilatory support has to be addressed as a matter of urgency whereas all concerned, including the patient, would much prefer to take decisions on this issue in a more thoughtful and proactive way. As always, when we have made appropriate measurements and know exactly what the situation is, it becomes much easier to practise high quality clinical medicine.

The report by Rigg and colleagues ${ }^{5}$ is again one of considerable interest, demonstrating that right phrenic nerve damage may be a far from rare event in patients being treated for malignant disease with repeated courses of cytotoxic drugs delivered through a right Hickman line. Thus, we now have yet another well documented cause of diaphragm dysfunction to add to the list! However, the overall clinical 
importance of hemidiaphragm paralysis is different from more diffuse diaphragm dysfunction. Isolated hemidiaphragm paralysis rarely causes substantial symptoms, and although maximum diaphragm strength is reduced to approximately half of normal values, overall respiratory muscle strength is less affected, vital capacity is approximately $75 \%$ of normal, and most patients are not breathless. ${ }^{6}$ A second important clinical point is that the diagnosis is not usually difficult to make. As in the cases reported by Rigg and colleagues, the paradoxical motion of the paralysed diaphragm can be easily appreciated by radiological screening or ultrasound scanning, and phrenic nerve stimulation on the affected side easily demonstrates that there is no movement of the hemidiaphragm, no EMG response, and (when measured) no transdiaphragmatic pressure response. However, the simple finding of a grossly elevated hemidiaphragm on the chest radiograph does not always signify paralysis and the tests carried out by Rigg et al are essential.

Dysfunction of the phrenic nerve/diaphragm muscle unit is not always all or nothing - for example, damage to a phrenic nerve root will cause weakness of the hemidiaphragm rather than paralysis. If an accurate assessment of hemidiaphragm function is required there is no good substitute for measuring Pdi in response to unilateral nerve stimulation. ${ }^{7}$

In summary, in recent years tests have become available that allow very precise measurement of respiratory muscle strength. In particular, the advent of magnetic stimulation has made phrenic nerve stimulation considerably easier for doctors and much more acceptable for the patient. If the question is whether or not there is diaphragm weakness, the answer is now relatively easily available.

1 Brander PE, Järvinen V, Lohela P, Salmi T. Bilateral diaphragmatic weakness: a late complication of radiotherapy. Thorax 1997;52:829-31.

2 Mier A, Brophy C, Moxham J, Green M. Assessment of diaphragm weakness. Am Rev Respir Dis 1988;137:877-83. 3 Polkey MI, Green M, Moxham J. Measurement of respiratory muscle strength. Thorax 1995;50:1131-5.

4 Laroche CM, Carroll N, Moxham J, Green M. Clinical significance of severe isolated diaphragm weakness. Am Rev Respir Dis 1988;138:862-6.

5 Rigg A, Hughes P, Lopez A, Filshie J, Cunningham D, Green $M$. Right phrenic nerve palsy as a complication of indwelling central venous catheters. Thorax 1997;52:831-3.

6 Laroche CM, Mier AK, Moxham J, Green M. Diaphragm strength in patients with recent hemidiaphragm paralysis. Thorax 1988;43:170-4.

7 Mills GH, Kyroussis D, Hamnegard CH, Wragg S, Moxham J, Green M. Unilateral magnetic stimulation of the phrenic nerve. Thorax 1995;50:1162-72.

\section{Bilateral diaphragmatic weakness: a late complication of radiotherapy}

\author{
Department of \\ Pulmonary Diseases \\ P E Brander \\ Department of \\ Clinical Physiology \\ $\mathrm{V}$ Järvinen \\ Department of \\ Radiology \\ P Lohela \\ Kiljava Hospital, FIN- \\ 05250 Kiljava, Finland \\ Division of Clinical \\ Neurophysiology, \\ Department of \\ Neurology, Helsinki \\ University Hospital, \\ FIN-00290, Helsinki, \\ Finland \\ T Salmi \\ Correspondence to: Dr P E \\ Brander, Scottish Nationa \\ Sleep Laboratory, Royal \\ Infirmary, Lauriston Place, \\ Edinburgh EH3 9YW, UK. \\ Received 21 March 1996 \\ Returned to authors \\ 12 August 1996 \\ Revised version received \\ 14 October 1996 \\ Accepted for publication \\ 4 November 1996
}

\author{
P E Brander, V Järvinen, P Lohela, \\ T Salmi
}

\begin{abstract}
Brachial plexus neuropathy is an unfortunate complication that sometimes follows radiotherapy to the axillary and supraclavicular regions. A patient is described who, 30 years after radiotherapy for Hodgkin's disease and more than 10 years after the development of radiationinduced bilateral brachial plexus neuropathy, presented with bilateral diaphragmatic weakness secondary to bilateral phrenic nerve weakness. Previous radiotherapy was the most probable cause of the condition.
\end{abstract}

(Thorax 1997;52:829-831)

Keywords: brachial plexus injury, diaphragmatic weakness, phrenic nerve paresis, radiation-induced neuropathy.

Brachial plexus neuropathy is a well recognised complication of radiotherapy to the axillary and supraclavicular lymph nodes, usually seen in association with radiotherapy for breast cancer and lymphomas. ${ }^{1}$ The incidence of disabling radiation-induced brachial plexus neuropathy has been as high as $19 \%$ in patients with breast cancer. ${ }^{2}$ Brachial plexus neuropathy may present several years after the radiotherapy; it typically shows progression over years and leads to permanent disability. The factors affecting the development of brachial plexus neuropathy include radiotherapy fields, total dose and duration of radiation, fractionation regimens, adjuvant therapy, and age of the patients. ${ }^{1-4} \mathrm{~A}$ total radiation dose of more than $60 \mathrm{~Gy}$ and a fraction size higher than $2 \mathrm{~Gy}$ have been shown to increase the risk of brachial plexus neuropathy. ${ }^{134}$ There is no effective treatment for the condition. As far as we know, phrenic nerve weakness associated with radiationinduced brachial plexus neuropathy has not previously been described.

\section{Case report}

A 67 year old moderately obese (weight $88 \mathrm{~kg}$, height $168 \mathrm{~cm}$ ) never-smoking woman was admitted in 1994 with severe orthopnoea which had developed gradually over a couple of years. During 1962-4 she had received four courses of radiotherapy to the supraclavicular and axillary regions and to the mediastinum because of Hodgkin's disease. The supraclavicular and axillary radiation dose was $100 \mathrm{~Gy}$ in total. After 1964 there had been no recurrence of Hodgkin's disease. In the 1970 s pain and muscular weakness developed in the patient's right, and later also left, arm. The symptoms progressed 
Table 1 Lung function data of the patient when seated and supine during wakefulness

\begin{tabular}{lcc}
\hline & Seated & Supine \\
\hline $\mathrm{PaO}_{2}(\mathrm{kPa})$ & 7.8 & 5.6 \\
$\mathrm{PaCO}_{2}(\mathrm{kPa})$ & 5.5 & 7.4 \\
$\mathrm{SaO}_{2}(\%)$ & 93 & 76 \\
$\mathrm{FEV}_{1}(1)$ & 1.1 & 0.4 \\
$\mathrm{FEV}_{1}(\%$ of predicted) & 44 & 16 \\
$\mathrm{FVC} \mathrm{(1)}$ & 1.1 & 0.5 \\
FVC (\% of predicted) & 36 & 14 \\
\hline
\end{tabular}

$\mathrm{PaO}_{2}=$ arterial oxygen tension; $\mathrm{PaCO}_{2}=$ arterial carbon dioxide tension; $\mathrm{SaO}_{2}=$ transcutaneous arterial oxygen saturation measured by pulse oximetry; $\mathrm{FEV}_{1}=$ forced expiratory volume in one second; FVC = forced vital capacity.

gradually over the years. In 1983 a neurological examination revealed severe muscle weakness and atrophy, paraesthesias, and decreased muscle stretch reflexes in both arms. Electroneuromyography (ENMG) showed a severe bilateral brachial plexus injury. Surgical treatment including breaking of bilateral perineural brachial plexus adhesions (neurolysis) and cutting of the left scalenus anterior muscle (scalenotomia) mitigated the pain but failed to improve the arm function.

On examination in 1994 the patient was dyspnoeic and cyanotic, and a paradoxical inspiratory inward movement of the abdominal wall was observed when she was lying on her back. There were no signs of heart failure. Spirometric tests showed impaired ventilatory function (table 1). Arterial blood gas analysis and measurement of arterial oxygen saturation by pulse oximetry $\left(\mathrm{SaO}_{2}\right)$, both performed with the patient sitting, showed mild hypoxaemia with normocapnia. Maximal inspiratory mouth pressure was low $(3 \mathrm{kPa})$ and relatively more reduced than maximal expiratory mouth pressure $(7 \mathrm{kPa})$. Change in position from sitting to supine was associated with a marked decrease in forced expiratory volume in one second $\left(\mathrm{FEV}_{1}\right)$ and forced vital capacity (FVC), a marked worsening of hypoxaemia, and development of hypercapnia (table 1). Nocturnal recording of airflow, respiratory and body movements, posture and $\mathrm{SaO}_{2}$ showed no signs suggestive of obstructive sleep apnoeas. ${ }^{5}$ The minimum value of nocturnal $\mathrm{SaO}_{2}$ was $50 \%$.

A high resolution computed tomographic (HRCT) scan of the chest showed normal lung parenchyma. Chest fluoroscopy revealed that the range of diaphragmatic movements during normal breathing was about $3 \mathrm{~cm}$ when the patient was standing. When she was lying on her back there was no diaphragmatic movement at all during quiet breathing. During sniffing a small paradoxical upward movement was observed. ENMG showed a chronic axonal bilateral brachial plexus injury which was more extensive than 10 years previously. It was associated with bilateral phrenic nerve weakness: the motor responses of the diaphragmatic muscle to the phrenic nerve stimulation were diminished and delayed (distal latencies $12.5-12.6 \mathrm{~ms}$, normal values less than $9 \mathrm{~ms}$ ). Phrenic nerve function was not investigated in 1983. ENMG showed no signs of generalised neuropathy, myopathy, or motor neurone disease.
The bilateral diaphragmatic weakness was treated by nasal ventilation with a bilevel positive airway pressure (BiPAP) ventilator ( $\mathrm{Re}$ spironics Inc, Murrysville, USA). During nasal ventilation the patient was able to lie on her back, and the $\mathrm{SaO}_{2}$ and arterial blood gas tensions remained normal in the supine position during both the daytime and at night. The patient has now been at home on nocturnal nasal ventilation for nearly two years, and the arterial blood gas tensions when the patient is sitting are normal.

\section{Discussion}

Both the clinical picture and the investigational findings in our patient were consistent with bilateral diaphragmatic weakness. Orthopnoea, inspiratory paradoxical motion of the abdominal wall in supine posture, fall in vital capacity by more than $50 \%$ when supine compared with the sitting position, reduced maximal inspiratory mouth pressure, and paradoxical or absent movement of the diaphragm when supine during inspiration (especially during sniffing) by chest fluoroscopy are all features compatible with bilateral diaphragmatic weakness. ${ }^{6}$ Unfortunately we were not able to measure the transdiaphragmatic pressures and so could not assess the actual strength of the diaphragm.

Bilateral diaphragmatic weakness is a rare condition and is usually associated with generalised neuromuscular diseases such as amyotrophic lateral sclerosis, multiple sclerosis, poliomyelitis, spinal muscular atrophy, myasthenia gravis, and muscular dystrophies. The phrenic nerves may become affected in various polyneuropathies, in neuralgic amyotrophy, by direct malignant invasion, in blunt chest traumas, and during open heart surgery. Systemic conditions such as connective tissue diseases, amyloidosis, and thyroid diseases may affect the diaphragmatic muscle. ${ }^{6}$ In our patient there was no evidence in the clinical history or in the neurological and neurophysiological examinations of generalised neuromuscular diseases (such as motor neurone disease and myasthenia gravis) or of other conditions leading to brachial plexus and phrenic nerve damage. During the last few years neither fluctuation nor progression in the neurological symptoms have been observed, nor have muscle groups other than those innervated by the brachial plexus or phrenic nerves been affected.

The diaphragmatic weakness in our patient was associated with bilateral phrenic nerve weakness and was probably caused by the radiotherapy given in the 1960s for Hodgkin's disease. She had received radiotherapy to the supraclavicular and axillary nodes in doses large enough to lead to severe bilateral brachial plexus neuropathy, which had been noticed more than 10 years previously. The phrenic nerve is anatomically located in the neck medially and close to the brachial plexus. It originates from nerve roots $\mathrm{C} 3-\mathrm{C} 5$, and the brachial plexus originates from nerve roots $\mathrm{C} 5$ T1. Thus, paresis of the phrenic nerve could be expected to occur frequently in association 
with brachial plexus injury. Ipsilateral hemidiaphragmatic paresis is often observed during interscalene brachial plexus blockade. ${ }^{7}$ However, diaphragmatic weakness has not been previously reported in association with radiation-induced brachial plexus neuropathy. In a recent report of a neurological follow up of 161 breast cancer patients after a disease-free interval of 50 (13-99) months, radiation-induced brachial plexus neuropathy was diagnosed in $5-9 \%$ of patients; ${ }^{4}$ no mention was made of phrenic nerve neuropathy. In addition, there have been no comments on diaphragmatic function in earlier studies with follow up times after radiotherapy of more than 30 years. ${ }^{128}$ Minor and, especially, unilateral diaphragmatic weakness may have been overlooked in previous studies. Radiation-induced brachial plexus neuropathy typically shows progression over years. ${ }^{8}$ This was also observed in our patient: according to the symptoms and to the ENMG the brachial plexus injury had progressed during the last 10 years. The phrenic nerves may also have been affected in their course through the thorax by the radiation given to the mediastinum. An isolated bilateral diaphragmatic paresis after mediastinal radiotherapy of Hodgkin's disease without brachial plexus involvement has recently been described. ${ }^{9}$

The ventilatory impairment in our patient may have been more pronounced than would have been expected to occur in isolated bilateral diaphragmatic weakness alone. ${ }^{10}$ In bilateral diaphragmatic weakness the role of other respiratory muscles is enhanced. Some of these muscles (the scaleni muscles) are innervated by the brachial plexus, so brachial plexus neuropathy decreases the function of these muscles. Respiratory muscle dysfunction in our patient was therefore more severe than in isolated diaphragmatic weakness. Previous left-sided scalenotomy and obesity may have further contributed to the ventilatory impairment. There were no findings of an associated lung disease.

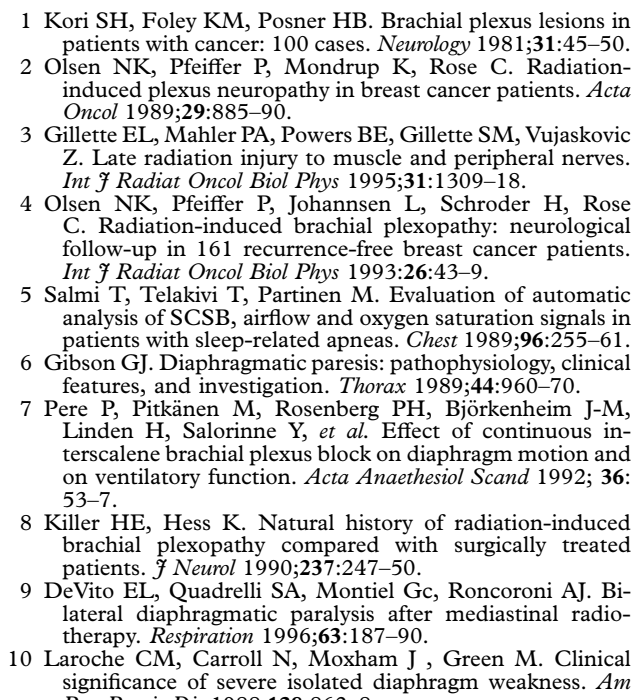
Rev Respir Dis 1988,138:862-8.
Cancer Research

Campaign

Section of Medicine,

Gastrointestinal Unit

A Rigg

D Cunningham

Department of

Radiology

A Lopez

Department of
Anaesthesia

Anaesthe

Royal Marsden

Hospital,

Downs Road, Sutton

Surrey SM2 5PT, UK

Respiratory Muscle

Laboratory,

Royal Brompton

Hospital,

Sydney Street,

London SW3 6NP, UK

P Hughes

M Green

Correspondence to: Dr D Cunningham.

Received 11 July 1996 Returned to authors 25 September 1996

Revised version received

23 October 1996

Accepted for publication

4 November 1996
Right phrenic nerve palsy as a complication of indwelling central venous catheters

Anne Rigg, Philip Hughes, Anthony Lopez, Jacqueline Filshie, David Cunningham, Malcolm Green

\footnotetext{
Abstract

Five cases are reported of patients who developed a raised right hemidiaphragm while an indwelling central venous catheter was in situ. The patients were being treated with protracted venous infusions of chemotherapy for colorectal carcinoma. All five patients had a chest radiograph following insertion of the Hickman line which showed normal diaphragmatic positions. A mean of 93 days later (range 55-134 days) elevation of the right hemidiaphragm was noted in these patients on repeat chest radiographs. Two of the patients had a right phrenic nerve palsy
}

demonstrated by magnetic stimulation of the nerve. The remaining three patients had paradoxical motion of the right hemidiaphragm on sonography, but were unable to undergo studies of phrenic nerve function before death from metastatic disease. It is suggested that right phrenic nerve palsy is a late complication of an indwelling central venous catheter.

(Thorax 1997;52:831-833)

Keywords: central venous catheter, phrenic nerve palsy, thrombosis, diaphragm.

Hickman lines are now integral to the administration of protracted venous infusions of 5-fluorouracil chemotherapy. During the past 12 months 650 Hickman lines have been inserted by anaesthetists using fluoroscopy at the Royal Marsden Hospital for this purpose. We report a late complication which occurred in five patients who had indwelling central venous catheters. All patients had a Hickman line inserted via the right subclavian vein using Seldinger's technique at the first attempt. All patients received $1 \mathrm{mg}$ warfarin from the day of the procedure, as is standard practice at this institution. The chest radiograph taken after the procedure showed normal diaphragmatic positions in all patients. 


\section{Case reports}

CASE 1

A 71 year old man was referred for adjuvant chemotherapy after resection of a Duke's B carcinoma of the rectum. A pretreatment chest radiograph showed hyperinflation of the upper lobes consistent with centrilobular emphysema, and a calcified granuloma of the right upper lobe. A computed tomographic (CT) scan of the thorax, abdomen and pelvis confirmed that no detectable metastatic disease was present.

Thirty one days after insertion of the Hickman line the patient complained of right shoulder pain. A chest radiograph and Duplex ultrasound scan of the neck and axillary veins were normal. The right shoulder pain persisted and three weeks later a repeat chest radiograph demonstrated elevation of the right hemidiaphragm. On this occasion the Duplex ultrasound scan showed a non-occluding thrombus in the right internal jugular vein, and sonography confirmed paradoxical motion of the right hemidiaphragm.

The Hickman line was removed and the patient therapeutically anticoagulated (international normalised ratio kept in the range of 2.5-3.5). Assessment of diaphragm function was performed using a magnetic stimulator (Magstim 200, The Magstim Co., Dyfed, UK) with a $43 \mathrm{~mm}$ coil positioned over the course of the phrenic nerve on the left and right sides of the neck in turn. Responses to maximal stimulation were measured as transdiaphragmatic twitch pressure recorded with balloon catheters positioned pernasally in the stomach and oesophagus. ${ }^{1}$ Transdiaphragmatic twitch pressure of $17 \mathrm{~cm} \mathrm{H}_{2} \mathrm{O}$ (normal $>7 \mathrm{~cm}$ $\mathrm{H}_{2} \mathrm{O}$ ) was elicited by stimulation of the left phrenic nerve with no response on the right, confirming unilateral phrenic nerve palsy.

Six months after removal of the Hickman line the patient remained disease free with no significant respiratory symptoms. The chest radiograph showed persistent elevation of the right hemidiaphragm.

CASE 2

A 67 year old woman received adjuvant infusional 5-fluorouracil chemotherapy following resection of a Duke's C adenocarcinoma of the rectum. There was no evidence of metastatic disease on a CT scan of the thorax, abdomen, and pelvis. The pretreatment chest radiograph was normal. The patient had no relevant past medical history. Seventy eight days after Hickman line insertion the patient complained of right shoulder pain and exertional dyspnoea. A chest radiograph at this time showed elevation of the right hemidiaphragm with a small right pleural effusion and mediastinal widening. A Duplex ultrasound scan of the right neck and axillary veins and peripheral upper limb venography revealed pericatheter thrombus in the superior vena cava.

The Hickman line was removed and the patient therapeutically anticoagulated. Magnetic stimulation of the phrenic nerves demonstrated a marked reduction in the response of the right phrenic nerve compared with the left compatible with a partial right phrenic nerve palsy (left phrenic nerve $11 \mathrm{~cm} \mathrm{H}_{2} \mathrm{O}$, right phrenic nerve $5 \mathrm{~cm} \mathrm{H}_{2} \mathrm{O}$ ).

At 12 month follow up the patient was disease free and had a persistently elevated right hemidiaphragm on chest radiography. Her dyspnoea and right shoulder pain had fully resolved.

CASE 3

A 60 year old man received adjuvant infusional 5-fluorouracil via a Hickman line following resection of a Duke's B adenocarcinoma of the colon. The initial chest radiograph was notable only for a calcified node at the left hilum. During treatment he developed new hepatic and splenic metastases at which point mitomycin $\mathrm{C}$ was added to the regimen. Three months after insertion of a Hickman line he complained of right shoulder and neck pain. The chest radiograph and Duplex ultrasound scan of the neck veins were normal. The patient's pain persisted and six weeks later he also described right shoulder pain on flushing the line. The chest radiograph now demonstrated an elevated right hemidiaphragm (123 days after insertion of the Hickman line). Ventilation-perfusion scans and bilateral upper limb venograms were normal. Sonography demonstrated paradoxical motion of the right hemidiaphragm. A CT scan confirmed that there was no metastatic disease within the mediastinum and that the liver metastases were stable and confined to segments V and VI of the right lobe of the liver As there was no demonstrable thrombus, the line remained in situ until the patient completed the treatment course and was then removed. On follow up chest radiographs the right hemidiaphragm remained elevated until the patient's death from metastatic liver disease one year later.

CASE 4

A 59 year old woman was referred for chemotherapy following a palliative triple bypass procedure for a carcinoma of the splenic flexure of the colon. She was treated with carboplatin and 5-fluorouracil was infused via a Hickman line. There was no evidence of mediastinal disease on a thoracic CT scan. Seventy three days later the patient presented with a severe headache and distended neck and arm veins. A venogram confirmed extensive thrombus extending from the right internal jugular vein to the right atrium. The chest radiograph demonstrated an elevated right hemidiaphragm. A ventilation-perfusion scan revealed no significant abnormality. The Hickman line was removed and the patient therapeutically anticoagulated. Despite anticoagulation with heparin, the patient developed further axillary vein thromboses. She was therefore treated with an infusion of streptokinase. The superior vena caval obstruction gradually resolved, but the right hemidiaphragm remained elevated. Sonography demonstrated paradoxical motion of the right hemidiaphragm. The patient died 
three months after the presentation of the superior vena caval obstruction from metastatic carcinoma of the colon.

CASE 5

A 49 year old man was found to have synchronous liver metastases at the time of resection of a rectal tumour. $\mathrm{He}$ received palliative infusional 5-fluorouracil chemotherapy. Over the four months after insertion of a Hickman line the patient complained of intermittent pain in the right shoulder region. Chest radiography, ventilation-perfusion scanning and Duplex ultrasound scans of the neck veins were normal. As pain persisted the Hickman line was eventually removed (134 days after insertion). A chest radiograph prior to removal showed a newly elevated right hemidiaphragm. Sonography confirmed paradoxical motion. The right hemidiaphragm remained elevated on subsequent follow up. The patient died one year later of metastatic disease, still with an elevated right hemidiaphragm.

\section{Discussion}

We hypothesise that the presence of the indwelling central venous catheter in these five patients was causally related to the subsequent development of a right phrenic nerve palsy. The two surviving patients have a proven right phrenic nerve palsy on formal nerve testing. The other three patients are deceased and therefore cannot be tested. However, all three had paradoxical motion of an elevated right hemidiaphragm on sonography suggesting that they had also incurred a palsy of the right phrenic nerve.

The right phrenic nerve arises from the third, fourth and fifth cervical nerve roots and passes posterior to the junction of the right internal jugular and subclavian veins as they form the right brachiocephalic vein. The right phrenic nerve then runs along the lateral aspect of the superior vena cava.

A right phrenic nerve palsy has been described as an immediate complication of central venous cannulation. ${ }^{2-5}$ In two of these cases ${ }^{23}$ there had been repeated attempts at cannulation of the right internal jugular or right subclavian veins, and it was suggested that the subsequent right phrenic nerve palsy was secondary to direct trauma. Medin et $a l^{4}$ described a transient right phrenic nerve paralysis due to local anaesthetic instilled at the time of cannulation of the right subclavian vein. In this case the chest radiograph taken immediately after the procedure showed an elevated right hemidiaphragm, but a repeat film one hour later was normal. Seaberg et al described a patient who received streptokinase thrombolysis after unsuccessful cannulation of the right subclavian vein in a patient who had suffered an acute myocardial infarction. ${ }^{5}$ The patient developed a right phrenic nerve palsy on the day of thrombolysis secondary to a right paratracheal haematoma subsequently demonstrated on a CT scan.

In our series of patients elevation of the right hemidiaphragm occurred 55-134 days (mean 93 days) after Hickman line insertion. A recent review suggested that $10 \%$ of oncological patients with a central venous catheter develop an associated thrombus, often exacerbated by a prothrombotic tendency. ${ }^{6}$ While these patients flushed their lines daily with $3 \mathrm{ml}$ of $100 \mathrm{U} / \mathrm{ml}$ heparin, none received oral anticoagulants. We hypothesise that venous wall inflammation due to the catheter alone, or in conjunction with thrombus, caused ischaemic damage to the vasa nervorum of the right phrenic nerve. It is unlikely that the palsies were related to the insertion procedure as they occurred much later. All the patients were receiving 5-fluorouracil chemotherapy via the Hickman line; although neuropathy is not a well recognised side effect of this drug, high concentrations of drug around the catheter tip could also contribute to nerve damage. Although unilateral diaphragm paralysis is clearly an uncommon complication, it appears to be well tolerated in the long term by our two surviving patients. All our patients noted an increase in exertional dyspnoea, as commonly occurs with hemidiaphragm paralysis $;^{7}$ however, none had formal measurement of pulmonary function prior to insertion of the Hickman line. Patients with cancer at the Royal Marsden Hospital with indwelling central venous catheters continue to receive prophylactic anticoagulation ( $1 \mathrm{mg}$ of warfarin per day) and this practice is under regular review.

We therefore conclude that right phrenic nerve dysfunction and hemidiaphragm paralysis is a late complication of central venous catheters. We propose that inflammation and thrombus development associated with the catheter cause damage to the phrenic nerve or its blood supply.

1 Mills GH, Kyroussis D, Hamnegard CH, Wragg S, Moxham $\mathrm{J}$, Green $M$. Unilateral stimulation of the phrenic nerve. Thorax 1995;50:1162-72.

2 Vest JV, Pereira M, Senior RM. Phrenic nerve injury associated with venipuncture of the internal jugular vein. Sociated with venip $1980 ; 78: 777-9$.

3 Hadeed HA, Braun TW. Paralysis of the hemidiaphragm as a complication of internal jugular vein cannulation: report of a case. $\mathcal{F}$ Oral Maxillofac Surg 1988;46:409-11.

4 Medin DL, Ognibene FP. Abnormal chest radiograph after central line placement. Respir Care 1993;38:125-7.

5 Seaberg DC, Generalovich T. Hemorrhagic compression of the phrenic nerve after streptokinase infusion. Am $\mathcal{F}$ Emerg Med 1989;7:185-6.

6 Eastridge BJ, Lefor AT. Complications of indwelling venous access devices in cancer patients. F Clin Oncol 1995;13 $233-8$

7 Laroche CM, Mier AK, Moxham J, Green M. Diaphragm strength in patients with recent hemidiaphragm paralysis Thorax 1988;43:170-4. 